\title{
Toledo Naloxone Outreach Program: Patient and Health Care Professional Student Education
}

\author{
Alex Petrak'; Luke Zona ${ }^{1}$; Jeni Ten Eyck'; Manish Karamchandani²; Tanvir Singh ${ }^{1}$ \\ ${ }^{1}$ College of Medicine and Life Sciences, University of Toledo, Toledo, $\mathrm{OH}$ \\ ${ }^{2}$ Department of Psychiatry and Behavioral Sciences, Medical University of South Carolina, Charleston, SC \\ Corresponding Author: Alex Petrak, 5859 Spring Hollow Drive, Toledo, OH 43615, (614) 716-9741, petrak.alex@gmail.com \\ Submitted October 28, 2020 Accepted April 7, 2021 Published June 21, 2021 https://doi.org/10.18061/ojph.v4i1.8072
}

\begin{abstract}
Background: In 2016, Ohio was home to 2 of the top 10 cities with opioid overdoses in the United States. Dayton ranked first, and Toledo held the tenth slot. In response to the opioid epidemic, the Toledo Naloxone Outreach Program (TNOP) was developed to provide naloxone to underserved patients at a student-run free clinic.

Methods: The TNOP takes place weekly at 2 locations in the greater Toledo area. Patients are asked to fill out an anonymous survey and given a brief training session by a health care professional student. Patients are then sent home with a free naloxone kit. Additionally, health care professional students were surveyed on their confidence of acquiring a substance use history and knowledge of local services for patients and family members of those experiencing addiction during the "train the trainer" event. The health care professional student survey utilized a 1-5 Likert scale and was analyzed using paired student $t$ tests.
\end{abstract}

Results: Survey results from patients indicate that a majority had not received naloxone prior to the training, indicating the service was expanding access to an otherwise underserved population. Results from the survey administered before and after health care professional student training sessions reflect a significant increase in confidence eliciting a substance abuse history and providing patients and/or family members with addiction resources in the community.

Conclusion: The TNOP has provided the community of Toledo with 2 additional outreach locations for receiving free naloxone kits with proper education. Currently, TNOP is in the process of expanding the outreach program to additional student-run free clinics in Ohio.

Keywords: Interdisciplinary teams; Pharmacy; Community health/engagement; Naloxone

\section{INTRODUCTION}

In 2018, Ohio ranked fifth in rates of age-adjusted drug overdose deaths. ${ }^{1}$ Within Ohio, Lucas County has experienced a steady rise in opioid overdose deaths from 2014-2019.2 Lucas County ranks seventh of 88 counties in Ohio regarding total drug overdoses from 2012-2020.3 If uninsured, the public can procure naloxone for $\$ 75$ from a variety of pharmacies and at no cost from the Toledo Lucas County Department of Health. The cost for naloxone can be covered depending on the individual's insurance. However, the need for readily available access to naloxone in underserved populations remains high. The American Society of Addiction Medicine
(ASAM) published a public statement supporting broadened community access to naloxone in populations with high risk for opioid overdose. ${ }^{4}$ Given the significant detrimental impact of opioid overdoses on the local community, the Addiction Medicine Club at the University of Toledo College of Medicine (UTCOM) created the Toledo Naloxone Outreach Program (TNOP) in August 2018. The TNOP was formed to combat the opioid epidemic at a local level by providing free naloxone training and kits to vulnerable populations. Additionally, the program aims to provide health care professional student education early on in training to prepare students to care for a population significantly affected by the opioid epidemic. 
The TNOP works in conjunction with the Lucas County Department of Health and Project DAWN (Deaths Avoided With Naloxone) through the Ohio Department of Health which provides grant funding for naloxone kits. Project DAWN is an Ohio-based nonprofit dedicated to providing naloxone training and distribution. To implement the program, TNOP partnered with Community Care Clinics (CCC), a student-run free clinic for the uninsured and unemployed in the greater Toledo area. As of September 2020, TNOP has aided in the training of over 1200 health care professional students on the signs of opioid overdose and how to act in the event of an overdose, including administering naloxone. This paper aims to show the impact of a student-run naloxone service on both professional health care student education and patient access to naloxone.

\section{METHODS}

\section{Setting}

The TNOP's naloxone service is provided each Thursday at Community Care Clinics' (CCC) main site and Mondays during CCC's Labre Traveling Clinic which operates at 2 street corners in downtown Toledo. An estimated 50 patients are screened per week for naloxone needs.

Health care professional student trainings took place in person on the campus of University of Toledo College of Medicine.

\section{Design}

The research utilized surveys for both the patient training and the health care professional student "train the trainer" event to determine outcomes of the naloxone service provided. For the patient survey, information was utilized to determine the need for a naloxone service in an underserved patient population.

From the "train the trainer" event, pre- and post-training surveys were utilized to determine if the event provided a significant increase in professional student knowledge of taking a substance use history and awareness of local addiction recovery resources.

For this analysis, data were collected between August 2018 and December 2019. The University of Toledo Institutional Review Board has granted exemption for this research.

\section{Participants}

During CCC's intake process, patients are asked if they or a family/ friend use opioids and are provided examples of opioids. If indicated, they are offered free naloxone training and kits during their clinic visit.

Interprofessional students, including students from the schools of social work, college of medicine, college of pharmacy, and college of nursing are eligible to receive training. Selection criteria were limited to students who had never received formal training on naloxone administration or education.

\section{Procedures}

The research was performed using information from 2 separate training protocols. The patient training process consisted of a 15minute private interactive session with a trained health care professional student regarding naloxone and its administration during an overdose as well as signs and symptoms of an opioid overdose. Prior to the training the patient was required to fill out the Project DAWN survey. After the training, patients were then sent home with a free naloxone kit. The kit contains 2 doses of $4 \mathrm{mg}$ intranasal naloxone, gloves, a face shield, and a card with rescue directions and local recovery service numbers. Patients with smartphones were encouraged to download the "NARCAN Now" smartphone app in case of emergency for rapid review of the materials.

Additionally, 2 types of health care professional training events were conducted. The first, "train the trainer," included details about pharmacokinetics, pharmacodynamics, and prescriber information. This is a standardized 1-hour in-person training session created by TNOP in conjunction with the local health department. Students were then eligible to train patients via the naloxone outreach program. The other type of student training was conducted through the Interprofessional Education Program (IPE) in conjunction with the Lucas County Health Department. This 20minute in-person training educated IPE students to personally respond to an opioid overdose situation using naloxone but did not qualify them to train patients. No survey was utilized during the IPE training and, therefore, is not included in data analysis.

Both types of training covered topics including trends in opioid usage and overdose in the Toledo area, signs and symptoms of narcotic overdose, the pharmacology of naloxone, and the appropriate steps to navigate a rescue scenario. Additionally, both types of training highlighted addiction recovery resources available locally and how to contact them.

\section{Measures/Outcomes}

Patients are required to fill out an anonymous 1-page survey from Project DAWN which includes 14 questions. This covers patient demographics, past overdose history, and naloxone use history. A copy of the survey can also be found in Appendix A. This valuable information is periodically returned to the Ohio Department of Health to evaluate the local need for naloxone and titrate funding.

Before and after the "train the trainer" session, students were asked to fill out a survey assessing their confidence in eliciting a substance use history from a patient or family member as well as their knowledge of local addiction recovery resources. Of the 100 students in attendance in the "train the trainer" session, 70 participants completed the pre-training survey and 35 completed the post-training survey. The discrepancy of pre- and post-training survey participation is due to some students leaving the event before filling out the post-training survey. 
A 1-5 Likert scale (1 = strongly disagree, 5 = strongly agree) was used for the health care professional student pre- and post-survey questions. The survey included 4 questions assessing confidence in eliciting a substance use history from patients and family members as well as their familiarity with addiction recovery resources in the Northwest Ohio area. A copy of this survey can be found in Appendix B.

\section{Statistical analysis}

Results were entered into a Microsoft Excel spreadsheet. Twotailed paired student $t$ tests were performed on the health care professional student survey responses. The Likert scale averages of pre- and post-training questions were compared. The data points from the 35 students that completed the pre-training survey but did not complete the post-training survey were not included in data analysis to allow for paired student $t$ tests to be conducted.

\section{RESULTS}

Project DAWN survey results were collected during the intake process from 35 participants after a patient expressed interest in naloxone training. While patients were required to fill out the survey to the best of their ability, not all patients answered each question in the Project DAWN survey. Many of the questions had a low response rate of less than 5 responses. The following 3 questions are included for discussion. When asked whether an individual has witnessed an overdose in the past, there was an equal distribution that answered yes $(11 / 22,50 \%)$, and no $(11 / 22,50 \%)$. Additionally, when asked the gender of the person who overdosed there was again an almost equal distribution (male: $12 / 26,46 \%$, female: $14 / 26,54 \%)$. For the vast majority, this was the first time patients received naloxone (yes: $27 / 30,90 \%$, no: 3/30, 10\%) (Table 1).

Approximately 550 IPE students attended a training session in 2018, and 550 IPE students attended in 2019. In 2018, 100 health care professional students participated in the "train the trainer" session.

Regarding the health care professional student survey, statistical significance was achieved when comparing pre-training question responses with their respective post-training responses. When asked about their confidence level to elicit a substance abuse history from someone experiencing addiction, there was an increase in the mean from 3.1 to $3.6(P<.05)$. Students reported an increase in the mean from 3.2 to 3.7 when asked about their confidence in eliciting this same abuse history from a family member of someone experiencing addiction $(P<.05)$. When asked about their confidence in relaying contact information of recovery services in the local area to those with a substance use disorder, students reported an increase in the mean of 2.6 to $4.2(P<.01)$ and to family members an increase of the mean from 2.5 to 4.4 post training $(P<.01)$ (Figure 1).

\section{DISCUSSION}

Through the efforts of TNOP, over 1200 health care professional students have been trained to administer naloxone. Over 100 of these students received further training to be eligible to train patients on the use of naloxone in an opioid overdose situation. Surveys indicate the training provided students with greater confidence to elicit a substance use history from patients and family members, as well as increased their knowledge of recovery resources available in the local community. This education aids the trainers during their interactions with patients at the free clinics and also during clinical rotations when encountering patients with a substance use disorder.

From mid-2018 through 2019, approximately 35 patients were trained on the use of naloxone and received free training kits. Data gathered from the Project DAWN surveys indicate that most patients received naloxone for the first time at these locations with 27 of 30 responding their first time receiving naloxone was at CCC while 11 of 22 reported witnessing an opioid overdose in the past. This suggests the services provided patients with naloxone when they otherwise would not have had it to prevent an overdose death. Reviewing data from Lucas County comparing June 2019 to June 2020 there has been a $64 \%$ increase in opioid overdoses (105 in June 2019 and 204 in June 2020) with a 32\% increase in fatalities (16 in June 2019 and 22 in June 2020). However, there has been a 62\% increase in naloxone given (95 in June 2019 and 180 in June 2020). ${ }^{5}$ We suspect that programs such as the TNOP may have played a role in preventing an increase in opioid overdose deaths relative to the increase in total overdoses.

The distribution of naloxone is of increasing importance in Lucas County and surrounding areas as detection of fentanyl was reported in $83 \%$ of opioid deaths (376/452 deaths) in 2019,3 a trend

Table 1. Patient Responses from Project DAWN Survey

\begin{tabular}{|llc|}
\hline $\begin{array}{l}\text { Have you witnessed an overdose in the past? } \\
\text { Gender of the individual who overdosed? }\end{array}$ & Male (12/26), 46\% & Female (14/26), 54\% \\
Is this the first time receiving naloxone? & Yes (27/30), $90 \%$ & No (3/30), 10\% \\
\hline
\end{tabular}




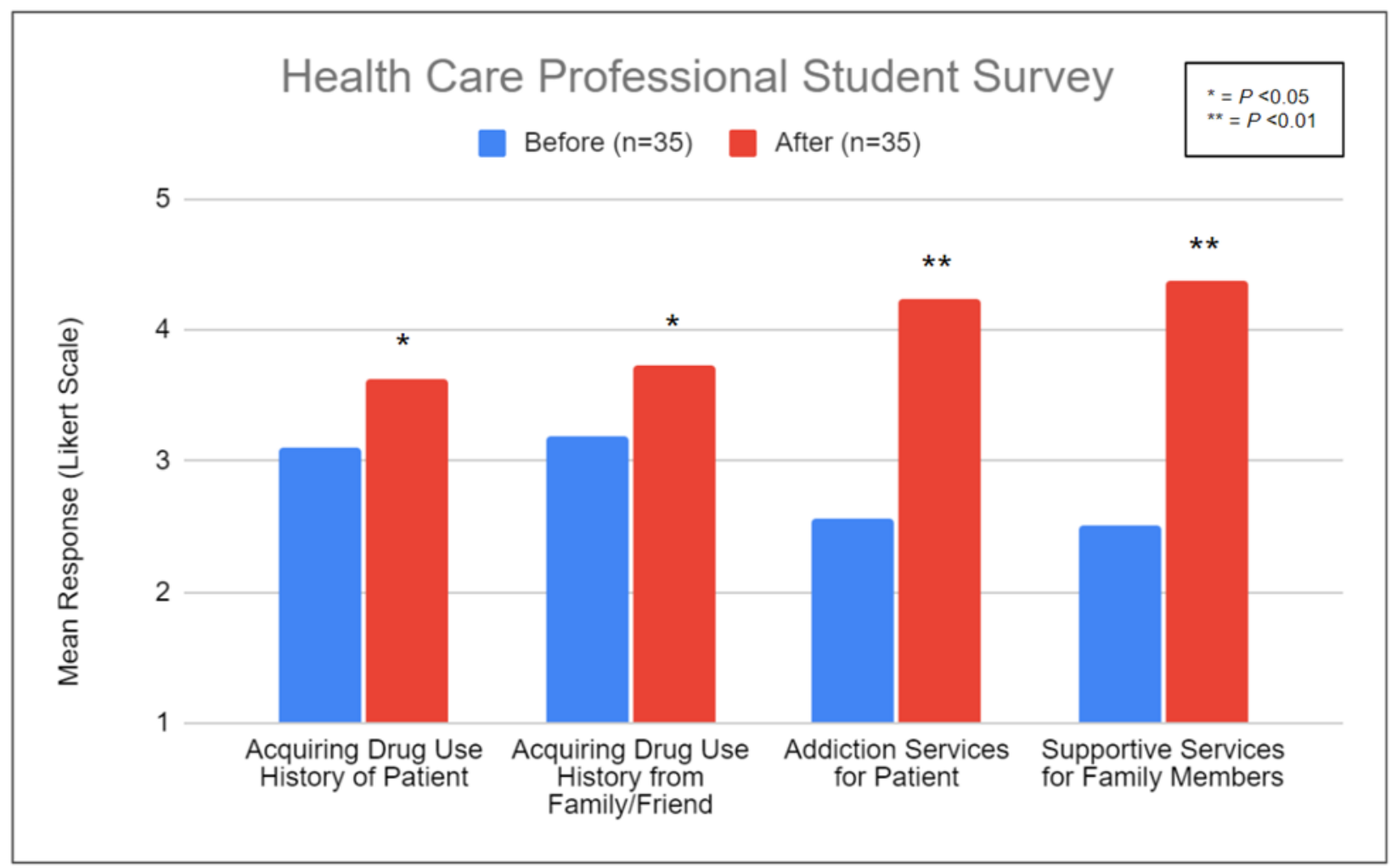

Figure 1. Health Care Professional Student Survey Outcomes

seen throughout the state of Ohio. ${ }^{6}$ Fentanyl is 50 to 100 times more potent than morphine or heroin, and early naloxone use is one of the most critical steps to prevent death in a fentanylinvolved overdose. ${ }^{7}$ Data from 2020 in Lucas County reveal that $74 \%$ of fatal overdoses occur in the person's home, and an additional $14 \%$ of deaths occur in a family/friend's house. ${ }^{8}$ With such a high percentage of fentanyl-involved opioid deaths, it is crucial to continue distributing naloxone to those at risk so that it is readily available in their home.

Limitations of this research include small sample sizes of students and patients surveyed. Some surveys were not filled out in their entirety by patients, thus the amount of people who answered each question varies compared to total patients surveyed. Additionally, there is a lack of follow-up data from patients after they received naloxone. Therefore, it is unknown if the patients went on to use their naloxone kit to prevent an opioid overdose death.

Future directions of the program include expanding TNOP to more locations within Toledo to allow a more diverse population of patients to have the opportunity to receive naloxone with highquality training, implementing an outcome survey to patients assessing their confidence in responding to an opioid overdose event pre- and post-training using a Likert scale, a formal analysis to confirm if a relationship exists between programs offering naloxone and a decrease in overdose fatalities, and creation of a replicable naloxone service for other health care professional schools in Ohio to use at their own institution in conjunction with their local health department and Project DAWN sites.

\section{PUBLIC HEALTH IMPLICATIONS}

The opioid epidemic has affected communities throughout the United States. While efforts have been put in place to mitigate the damage, opioids accounted for $83.7 \%$ of all unintentional drug overdoses in 2019 within Ohio. ${ }^{1}$ A 2017 report from the White House explains that this epidemic has cost the country $2.8 \%$ of the total GDP, which is roughly $\$ 500$ billion in $2015.9^{9}$ This epidemic is unique in that all demographics are affected. It is therefore imperative that all health care team workers in the United States are equipped with the knowledge to identify, support, and treat patients who are affected. When properly executed, health care professional student programs can combat the opioid epidemic at a local level. This study highlights the need for the service in the community and the augmentation of professional student education of addiction to aid in providing high quality patient care. An extra emphasis here is placed on all members of the health care team, as stigma in seeking help remains one of the greatest barriers in this fight.

\section{ACKNOWLEDGMENTS}

We would like to thank the University of Toledo College of Medicine and Life Sciences, Community Care Clinic, Lucas County Health Department, Ohio Department of Health, and Project DAWN. 


\section{REFERENCES}

1. Hedegaard H, Miniño AM, Warner M. Drug overdose deaths in the United States, 1999-2018. NCHS Data Brief, no 356. Hyattsville, MD: National Center for Health Statistics; 2020. Accessed October 2020.

https://www.cdc.gov/nchs/products/databriefs/db356.htm

2. Forney RB, Shinaver R. 2014-2019 Opioid Deaths. Lucas County Coroner Toxicology Laboratory: Lucas County; 2020. Accessed October 2020.

3. Summit County Public Health. Estimated Drug Overdoses, State of Ohio, Counties, and Zip Codes, 2012-2020. Columbus, Ohio: Ohio Department of Health; 2020. Accessed October 2020.

https://app.powerbi.com/view?r=eyJrIjoiZTkxZDAwOGQtNjYyMS00 ZTQ5LTg0OTctOTNiMzMwMGE0MTE2IiwidCI6ImJiMWI0YjU2LTQ 4N2EtNGIyMy04YTI0LWEzYWVmNjVlMTFmZiIsImMiOjF9

4. American Society of Addiction Medicine. Public Policy Statement on the Use of Naloxone for the Prevention of Opioid Overdose Deaths. Chevy Chase, MD; 2016. Accessed October 2020. https://www.asam.org/docs/default-source/public-policystatements/use-of-naloxone-for-the-prevention-of-opioid-overdosedeaths-final.pdf

5. Toledo Fire and Rescue. OD Response. Toledo, Ohio: Lucas County Health Department; 2020. Accessed October 2020.

6. Ohio Department of Health. Drug Overdose. Columbus, Ohio: Ohio Department of Health; 2021; Accessed January 2021 https://odh.ohio.gov/wps/portal/gov/odh/know-our-programs/ violence-injury-prevention-program/Drug-overdose/

7. Center for Disease Control. Occupational Exposure to Emergency Responders - NIOSH Workplace Safety \& Health Topics. Atlanta, GA: CDC; 2016. Accessed October 2020.

https://www.cdc.gov/niosh/topics/fentanyl/

8. Lucas County Health Department. Lucas County Drug Overdose Fatality Quarter 4. Toledo, OH: Lucas County Health Department; 2020. Accessed October 2020.

9. The Council of Economic Advisers. The Underestimated Cost of the Opioid Crisis. Washington D.C; 2017. Accessed February 2021. https://trumpwhitehouse.archives.gov/sites/whitehouse.gov/files/ images/The $\% 20$ Underestimated $\% 20$ Cost $\% 20$ of $\% 20$ the $\% 200$ pioid $\%$ 20Crisis.pdf 


\section{NALOXONE INTAKE FORM}

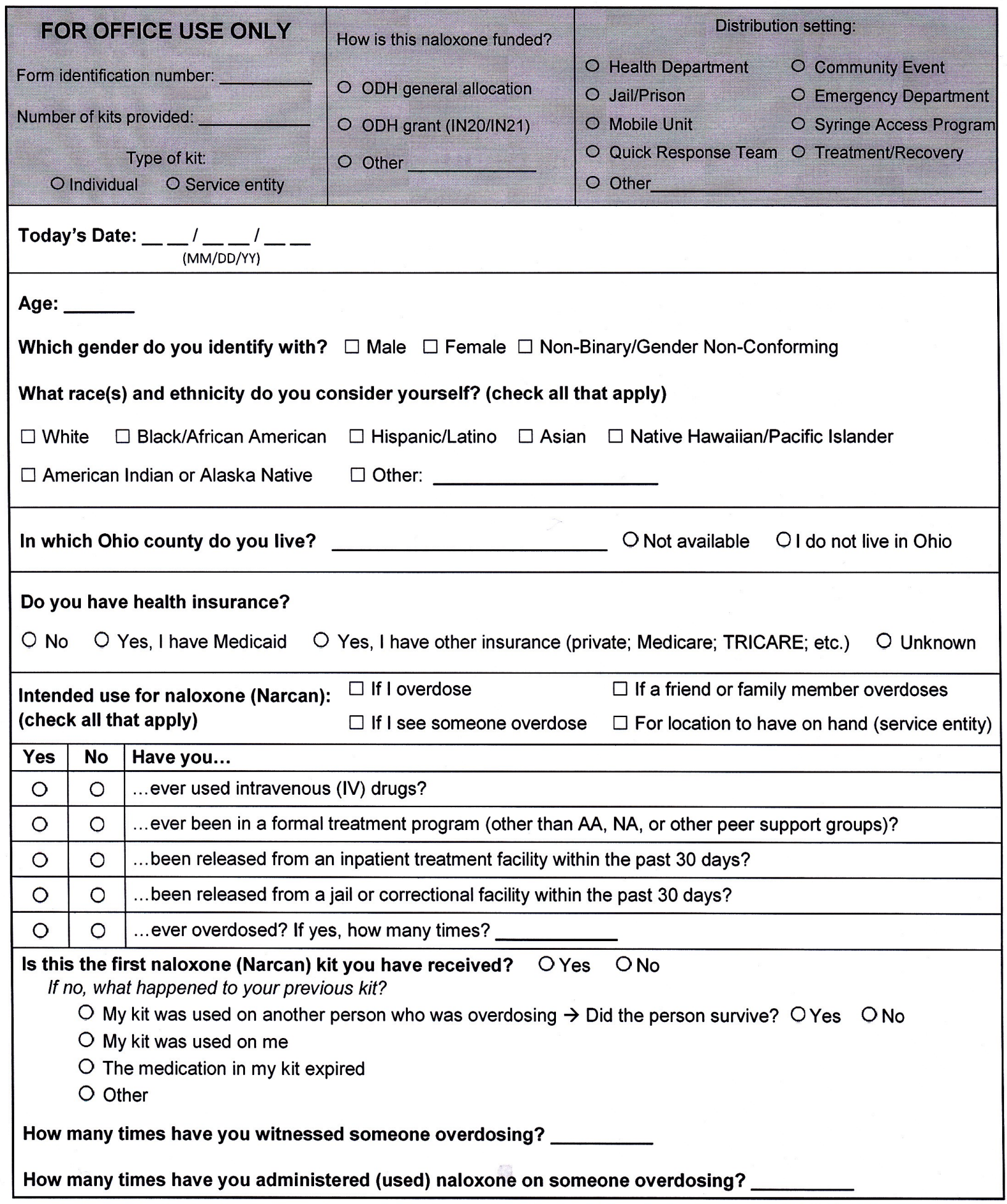


Date:

\section{Naloxone Outreach Program Healthcare Professional Student Survey}

1. I am confident I could elicit a substance abuse history from someone with addiction (Circle One)

\section{Strongly Disagree}

Disagree

Neither agree nor disagree

Agree

Strongly Agree

2. I am confident I could eliciting a substance abuse history from a family member or friend of someone with addiction (Circle One)

Strongly Disagree

Disagree

Neither agree nor disagree

Agree

Strongly Agree

3. I am confident I could provide contact information to those seeking addiction recovery programs in northwest ohio (Circle One)

Strongly Disagree

Disagree

Neither agree nor disagree

Agree

Strongly Agree

4. I am confident I could provide contact information to family members of those suffering from addiction in northwest ohio (Circle One)

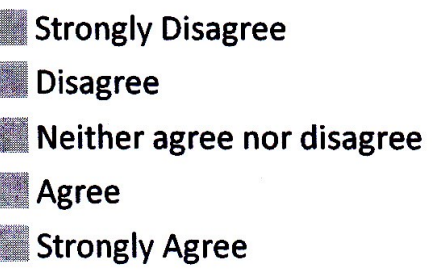

